\title{
Post-Traumatic Stress Disorder and Burnout in Healthcare Professionals During the SARS- CoV-2 Pandemic: A Cross-Sectional Study
}

\author{
Ioannis Ilias ${ }^{1 *}$, Vassiliki Mantziou2, Efstratios Vamvakas², Efstathia Kampisiouli2, Maria \\ Theodorakopoulou², Chariklia Vrettou², Evangelia Douka², Alice G. Vassiliou², Stylianos \\ Orfanos², Anastasia Kotanidou², Ioanna Dimopoulou ${ }^{2}$ \\ 1 Elena Venizelou Hospital, Athens, Greece \\ 2 National and Kapodistrian University of Athens
}

\begin{abstract}
Introduction: Healthcare professionals who are directly involved in the diagnosis, treatment, and general care of patients with SARS-CoV-2 are at risk of developing adverse psychological reactions. A cross-sectional study of healthcare professionals aimed to determine the impact of the SARS-CoV-2 pandemic on the mental health of healthcare professionals in two of the largest referral hospitals in Athens, Greece. Methods: The study was conducted in the two largest SARS-CoV-2 referral hospitals in Athens, Greece. An assessment and the interrelationship of post-traumatic stress disorder, using the Impact of Event Scale-Revised [IES-R]) and burnout, using the Maslach Burnout Inventory [MBI]) was carried out. Results: A total of 162 subjects were enrolled in the study. Fifty-six (35\%) had an IES-R score $>33$, suggesting post-traumatic stress disorder. Forty-nine (30\%) had an MBI score $>27$. Seventy-five (46\%) had a personal accomplishment score of $<33$ and $46(28 \%)$ had a depersonalization score $>10$. Stepwise backward logistic regression revealed that the only independent variable that was retained regarding the presence of post-traumatic stress disorder was the emotional exhaustion score of the MBI (at a cut-off of 24 in this scale, the $95 \% \mathrm{Cl}$ of the odds ratio for the presence of post-traumatic stress disorder was 1.077-1.173). Conclusions: In this sample of first-line Greek healthcare professionals against SARS-CoV-2, most of them were proven to be quite resilient to this challenge. One-third of them had post-traumatic stress disorder, which depended on their degree of emotional exhaustion. Healthcare professionals, as represented by this study, performed their duties without feeling helpless and developing adverse psychological reactions.
\end{abstract}

Keywords: COVID-19, SARS-CoV-2, myocarditis, cardiac injury, extracorporeal membrane oxygenation, acute respiratory distress syndrome (ARDS), cardiogenic shock

Received: 8 July 2020 / Accepted: 31 October 2020

\section{INTRODUCTION}

Healthcare professionals who are directly involved in the diagnosis, treatment and general care of patients with SARS-CoV-2 are at risk of developing adverse psychological reactions. Anxiety, depression, mood disorders, insomnia, burnout, substance abuse, distress, post-traumatic stress disorder, irritability, fatigue or even increased suicidal tendency have all been reported [1-5].

Healthcare professionals may feel unqualified and ill-equipped to carry out clinical interventions in patients infected with this little known new virus, and no well-established clinical protocols or treatments are available. The fear of autoinoculation, burnout, the possibility of spreading the virus to their families, friends or colleagues and the overwhelming workload, may contribute to the mental burden of healthcare professionals [1-5].

Post-traumatic stress disorder can occur in people who have experienced or witnessed a traumatic event $[6,7]$. Most people recover quickly; for some, however, the effects can be long-lasting. The characteristic core of the disorder includes the distressing oscillation between intrusion, avoidance and hyperarousal. Intrusion involves re-experiencing the trauma, flashbacks, and nightmares. Avoidance of places, people, and activities that are reminders of the trauma are prominent. In 
post-traumatic stress disorder, being aroused, difficulty in sleeping and concentrating, being easily irritated or angered, are characteristic. Burnout is defined as a long-term reaction to occupational stress [8]. It has traditionally been conceptualized as encompassing three dimensions, emotional exhaustion, depersonalization, and lack of personal accomplishment. Post-traumatic stress disorder and burnout are significant problems experienced by healthcare professionals [9-12]. Persistent or chronic occupational burnout is considered to be a predictor of post-traumatic stress disorder [12].

The present study aimed to ascertain the impact of the SARS-CoV-2 pandemic on the mental health of healthcare professionals in two of the largest referral hospitals in Athens, Greece. The prevalence of posttraumatic stress disorder and burnout were recorded, and the relationship between the two was assessed.

\section{METHODS}

This double-centre study was a cross-sectional survey conducted between Monday the 1st of June and Sunday the $7^{\text {th }}$ of June 2020 at the "Evangelismos" and "Attikon" General Hospitals, Athens, Greece, two of the largest SARS-CoV-2 referral hospitals in the country.

The participants ( $\mathrm{n}: 239)$ were contacted face-to-face by the authors during morning briefings in their workplace (convenience sampling) and were asked to fill out, at their ease, but on-site, an anonymized multipart questionnaire. All the participants were healthcare professionals, physicians, nurses and technicians employed in these two referral hospitals.

Written informed consent was obtained on a form attached to the questionnaire package. The Ethics Committee at the host site approved the study (No 195/21.05.2020).

\section{Measuring Methods}

Demographic section: This section contained five questions referring to demographic and professional characteristics including sex, age, marital status, having children or living alone. The professional status was also addressed by two questions relevant to education qualifications, such type of original university degree, medical or other, Master's or PhD degrees, high school diploma, technical educational institute degree or other.

The section also included one question relating to the reasons for having selected their appointment. (Appendix 1; Translated from Modern Greek to English).
Professional activity section: This section addressed the professional standing (physician, nurse, or technician), workplace department (Intensive Care Unit [ICU], emergency department, High Dependency Unit [HDU] or ICU-affiliated laboratory) and months of professional experience (overall professional experience, working in the same hospital or the same department. (Appendix 1; Translated from Modern Greek to English).

Post-traumatic stress disorder section: The Impact of Event Scale-Revised (IES-R) was used [13, 14]. The Greek version of IES-R is available and has been validated [15].

Burnout section: The Maslach Burnout Inventory (MBI) was used [16]. BO is detected using cut-off scores of high emotional exhaustion $(\geq 27)$, high depersonalization $(\geq 10)$, and low personal accomplishment $(\leq 33)$, based on normative data. The Greek version of the MBI is available and has been validated [17].

\section{Statistical analysis}

The categorical variables were described by absolute numbers (n) and percentages (\%). Continuous variables were described by mean (SD) or median values and 25th-75th percentile if their distribution was asymmetric. Between groups (by profession) analysis was performed with analysis of variance (ANOVA) or the Kruskal-Wallis test. Further analysis was done with stepwise backward logistic regression using PTSD as the dependent variable (subjects with IES-R questionnaire score $>33$ considered to denote PTSD) and the following independent variables: age, sex, education level, living status (alone or not), marital status, children, time in months of work (total professional work experience, working in the same hospital and working in the same department), current hospital department or unit, profession (physician, nurse or other) and the MBI scores (depersonalization, personal accomplishment and emotional exhaustion subscales).

Additionally, following the stepwise backward logistic regression analysis, a three (physician/nurse/technician) by two (high or low emotional exhaustion score) between-subjects factorial ANOVA was undertaken, which compared PTSD (using the Impact Event Scale scores) according to the profession and emotional exhaustion status. A Receiver Operating Characteristic (ROC) curve for the emotional exhaustion score of the MBI was plotted using PTSD as the classification variable. The optimal cut-off value for predicting PTSD was calculated as the point with the most significant com- 
16 The Journal of Critical Care Medicine 2021;7(1)

bined sensitivity and specificity. Statistical significance was set at $\alpha<0.05$. The MedCalc statistical data software (v.14.8.1, 2014, MedCalc Software Ltd, Oostende, Belgium), was used for analysis.

\section{RESULTS}

Questionnaires were handed to 239 healthcare professionals, 162 responded (Response rate of approximately $68 \%$; Table 1 ).

Subjects were working in the profession for a median of 158 months, in the same hospital for 75 months and in the same department for 36 months. The reasons why they chose their profession were:

- To help people ( $n=103)$, being influenced by their environment $(\mathrm{n}=30)$,

Table 1. Demographic characteristics and professional details of the subjects

\section{Variables}

Gender

Men

Women

Age (yrs), mean(SD)

Marital status

Married

Not married

Separated, divorced, widow/er

Children

Yes

No

Living alone

Yes

No

Education

High school graduate

Technological educational

institution graduate

University graduate

University Degree, Master's Diploma,

PhD

Profession

Physician

Nurse

Technician

Department

Intensive Care Unit

Emergency department

High Dependency Unit

Laburnout ratory
N (\%)

37 (23)

125 (77)

39(9)

$88(54)$

$64(40)$

$10(6)$

$85(52)$

$31(48)$

$31(20)$

$131(80)$

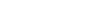

$26(16)$

59 (36)

77 (48)

$43(27)$

$102(63)$

$17(10)$

95 (59)

17 (10)

34 (20)

$16(11)$
- By chance $(n=26)$

- To attain a job $(n=66)$.

The median value for IES-R was 23 (IQR: 12-40) and 56 of the 162 subjects (35\%) had abnormal scores, i.e. IES-R > 33, suggesting post-traumatic stress disorder. The median intrusion sub-score was 8 (IQR: 4-16), the avoidance sub-score was 8 (IQR: 4-16), and the hyperarousal sub-score was 6 (IQR: 2-12).

The MBI emotional exhaustion score was 21(11), the personal accomplishment score was 33(10), and the median depersonalization score was 6 (IQR: 2-11). In $49 / 162$ subjects (30\%) the emotional exhaustion score was abnormal (i.e. $>27$ ), in $75 / 162$ subjects $(46 \%)$ the personal accomplishment score was low (i.e. $<33$ ) and in $46 / 162$ subjects $(28 \%)$ the depersonalization score was high (i.e. $>10$ ).

Physicians had lower post-traumatic stress disorder scores compared to nursing staff and technicians and also had lower emotional exhaustion scores compared to nursing staff (Table 2).

Stepwise backward logistic regression revealed that the only independent variable that was retained regarding the presence of post-traumatic stress disorder was the emotional exhaustion score of the MBI (at a cut-off of 24 in this scale, the $95 \%$ CI of the odds ratio for the presence of post-traumatic stress disorder was: 1.0771.173 , beta: 0.117 , chi-square: $42.163, \mathrm{p}<0.001$; sensitivity was $69 \%$ and specificity was $80 \%$ ).

Table 2. Comparisons between professional groups; means (SD) or 25thpercentile/median/75thpercentile

IES-R (Post-traumatic stress disorder)

score

Physicians

23.51(16.15)

Nurses

$30.04(18.96)^{*}$

Technicians

$32.23(26.268)^{*}$

$\mathrm{MBI}$ emotional exhaustion score

Physicians

$16.69(9.41)$

Nurses

$22.78(11.20)^{*}$

Technicians

19.29(13.45)

$\mathrm{MBI}$ personal accomplishment score

Physicians

31.46(11.34)

Nurses

$33.54(8.58)$

Technicians

$35.05(12.18)$

$\mathrm{MBI}$ depersonalization score

Physicians

$2.00 / 6.00 / 10.75$

Nurses

2.00/6.00/13.00

Technicians

$0.00 / 3.00 / 9.50$ 
In the $3 \times 2$ factorial ANOVA, a significant main effect for emotional exhaustion status was found $[\mathrm{F}(1$, 162): 35.984, $\mathrm{p}<0.001]$. Subjects who had high emotional exhaustion score also had high post-traumatic stress disorder scores (42.360(20.03) vs 19.819 (15.55), for those with low emotional exhaustion score). A less significant main effect was noted for profession $[\mathrm{F}(2$, 162):3.602, p: 0.036]; the mean(SD) post-traumatic stress disorder score for physicians at 23.513(16.14)was lower compared to that of nurses $(30.039 \pm 18.957)$ or technicians (32.230 \pm 26.258$)$. The interaction of emotional exhaustion status and profession was not significant [F (2,162):0.733, p:0.482].

The corresponding ROC plot for the emotional exhaustion score of the MBI vs presence of post-traumatic stress disorder had an area of 0.789 (Figure 1).

\section{DISCUSSION}

In the present study, we investigated the presence or not of burnout and post-traumatic stress disorder and the factors that affected them in healthcare professionals working in two SARS-CoV-2 reference hospitals in Athens, Greece. Physicians had lower post-traumatic stress disorder scores compared to nursing staff and technicians. Physicians also had lower emotional exhaustion scores compared to nursing staff. These findings are in accord with earlier studies. Similar results have also been observed during epidemics and pandemics) $[18,19]$.

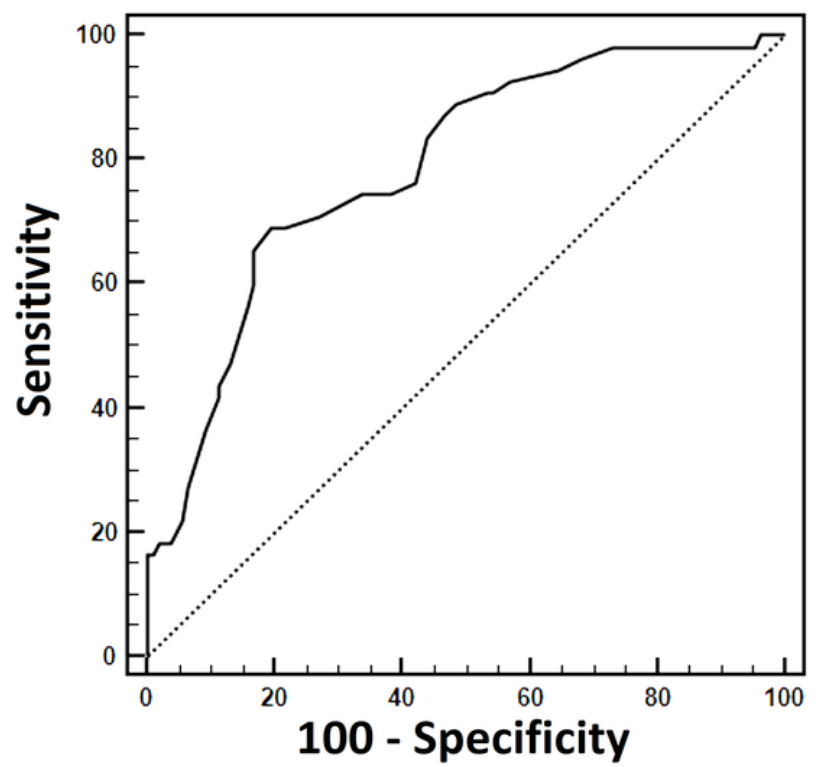

Fig. 1. Receiver Operating Characteristic plot of the emotional exhaustion score of the MBI vs presence of posttraumatic stress disorder; with a cut-off of 24 in this scale, sensitivity was $69 \%$, and specificity was $80 \%$.
According to the findings of the present study, the presence of post-traumatic stress disorder was closely related to the presence of burnout, and especially to the subjects' score in the emotional exhaustion scale of the MBI.

Several studies have shown that there is burnout in ICU staff, employees in hazardous or highly specialized occupations, those with high expectations for social work, such as nurses and physicians, and those occupations with a high degree of responsibility/accountability [20]. Emotional exhaustion is the initial and most important dimension of burnout due to increased and prolonged exposure to stress in the workplace.

It is essential to understand the relationship between chronic stress and burnout in conjunction with the conditions in Greek Public Health System Hospitals and the expectations of healthcare professionals. Emotional exhaustion in healthcare professionals, a feature of burnout as a result of chronic stress, can be attributed to the working conditions in Greek Public Health System Hospitals. The long-running economic crisis has led to a shortage of medical and nursing staff, limited resources, and the inability to provide immediate services to patients [21]. Thirty to $50 \%$ of Greek healthcare professionals have some degree of burnout [21]. Incidentally, in the current study sample, the few healthcare professionals who had chosen their profession by chance, were more apt to show emotional exhaustion as a feature of burnout (with a $95 \%$ CI of the odds ratio 1.471-8.946, beta: 1.262 , chi-square: 8.003 , $\mathrm{p}<0.001$ ); this is understandable, regardless of the current pandemic.

The observed rate of post-traumatic stress disorder in the ccurrent sample of healthcare professionals was not high. This may be explained by the fact that the pandemic in Greece did not explode with a large number of cases, and few patients needed hospitalization in wards or ICUs [22]. The healthcare professionals of the two hospitals were called upon to deal with the pandemic, with staff transfers from clinics and by manning newly created ICUs.

Fortunately, in Greece, up to the time of writing, healthcare professionals were not confronted with dire situations, such as ward and ICU bed shortages or dilemmas regarding the survival of patients, as timely public health measures and a generalized lockdown helped to prevent the spread of SARS-CoV-2 in Greece.

Studies have shown a strong positive correlation between burnout and post-traumatic stress disorder. This 
is likely to be initiated by a traumatic event, mainly if the process of emotional exhaustion is active $[23,24]$.

The onset of the SARS-CoV-2 pandemic is traumatic for healthcare professionals; it is an unknown novel pathogen for humans, with healthcare professionals worldwide having no previous experience of it.

Existing chronic stress and emotional exhaustion in the workplace, affect the degree of stress management imposed by the pandemic and consequently, the occurrence of post-traumatic stress disorder $[25,26]$.

Evidently, in Greece, healthcare professionals had the opportunity to care for patients with dignity, without feeling helpless, as has been reported for healthcare professionals in Italy [27], or of losing control of the situation. Nevertheless, it is possible that previous emotional exhaustion in combination with personal characteristics favoured the appearance of post-traumatic stress disorder.

Two factors were crucial for the correct initial course of the SARS-CoV-2 pandemic in Greece, with, apparently, moderate psychological effect on healthcare professionals. These were the prompt nation-wide lockdown, leading to a relatively small number of cases, and the functioning of the Greek National Health Service. Gowns, antiseptics, masks, gloves and other protective gear were promptly provided in anticipation of the pandemic to healthcare professionals. Healthcare professionals were transferred from smaller hospitals to SARS-CoV-2 referral hospitals. The number of ICU beds were doubled by upgrading HDUs to ICUs. PostAnaesthesia Care Units also were upgraded to ICUs. All scheduled elective surgical operations and outpatient clinics were cancelled. Healthcare professionals were given additional remuneration from the state as well as from private foundations. This resulted in the availability of beds for all SARS-CoV-2 patients that needed to be admitted to a ward or ICU.

Contrary to other studies [8], the present study did not find significant correlations between post-traumatic stress disorder, gender, age, marital status and the existence of children. The unequal distributions of these variables, the majority of those who answered the questionnaires were currently married or had been married, had children and did not live alone, may have played a role in this effect[29].

Though the sample size of the study was relatively small, it was not different from the sample size of analogous studies in Greece [30-32]. Furthermore, using the reported epidemiology of burnout as a reference level, with a prevalence of approximately $45 \%$ in healthcare professionals in Greece [21], and alpha $=0.10$, the posthoc power of this study was calculated to be $86 \%$.

\section{CONCLUSION}

In many healthcare systems that were overwhelmed by COVID-19, the number of hospital beds and more in particular intensive care unit beds became insufficient. Under such dire conditions, HSPs were faced with long working hours, the need to deal with the necessity to implement strict measures of personal protection against the virus and to supress moral dilemmas such as decisions on choice of patients to intubate and put on a ventilator, leading to "moral injury". These problems, along with the fear of the unknown disease and the unpredictable evolution of the pandemic, created a unique situation leading to post-traumatic stress disorder. Nevertheless, in well-prepared healthcare systems that were not overwhelmed by COVID-19, the effects on healthcare professionals are not so severe.

In Greece, the public healthcare system coped well with the situation. Hospital beds, and ICU beds, in particular, were sufficient in the spring of 2020 and healthcare professionals, employing "shunting" from different departments and hospitals were not overworked.

In the current sample of first-line Greek healthcare professionals dealing with SARS-CoV-2, most of them proved to be quite resilient to the challenge. One-third of them had post-traumatic stress disorder, which depended on their degree of emotional exhaustion.

The conclusion reached from the current study is that the current pandemic per se is not the only reason leading to burnout. Rather, prior chronic work stress over extended periods, workplace experiences and conditions modulate the effect of perceived stress regarding post-traumatic stress disorder.

\section{- CONFLICT OF INTEREST}

None to declare.

\section{REFERENCES}

1. Yin $Q$, Sun $Z$, Liu $T$, et al. Post-traumatic stress symptoms of health care workers during the coronavirus disease 2019. Clin Psychol Psychother. 2020;27:384-95.

2. Wang YX, Guo HT, Du XW, Song W, Lu C, Hao WN. Factors 
Available online at: www.jccm.ro

associated with post-traumatic stress disorder of nurses exposed to coronavirus disease 2019 in China. Medicine (Baltimore). 2020;99:e20965.

3. Vindegaard N, Eriksen Benros M. COVID-19 pandemic and mental health consequences: Systematic review of the current evidence. Brain Behav Immun. 2020.

4. Dutheil F, Mondillon L, Navel V. POST-TRAUMATIC STRESS DISORDER as the second tsunami of the SARS-Cov-2 pandemic. Psychol Med. 2020:1-2.

5. Chew NWS, Lee GKH, Tan BYQ, et al. A multinational, multicentre study on the psychological outcomes and associated physical symptoms amongst healthcare workers during COVID-19 outbreak. Brain Behav Immun. 2020;88:559-65.

6. Howlett JR, Stein MB. Prevention of Trauma and StressorRelated Disorders: A Review. Neuropsychopharmacology. 2016;41:357-69.

7. Sareen J. Posttraumatic stress disorder in adults: impact, comorbidity, risk factors, and treatment. Can J Psychiatry. 2014;59:460-7.

8. Thiery C. Qu'est-ce que le burnout ? Rev Prat. 2018;68:892-6.

9. McManus IC, Winder BC, Gordon D. The causal links between stress and burnout in a longitudinal study of UK doctors. Lancet. 2002;359:2089-90

10. Tselebis A, Bratis D, Karkanias A, et al. Associations on dimensions of burnout and family support for a sample of Greek nurses. Psychol Rep. 2008;103:63-6.

11. Bratis D, Tselebis A, Sikaras C, et al. Alexithymia and its association with burnout, depression and family support among Greek nursing staff. Hum Resour Health. 2009; 7:72.

12. Mealer M, Burnham EL, Goode CJ, Rothbaum B, Moss M. The prevalence and impact of post-traumatic stress disorder and burnout syndrome in nurses. Depress Anxiety. 2009;26:111826.

13. Creamer M, Bell R, Failla S. Psychometric properties of the Impact of Event Scale-Revised. Behav Res Ther. 2003;41:148996.

14. Hyer K, Brown LM. The Impact of Event Scale-Revised: a quick measure of a patient's response to trauma. Am J Nurs. 2008;108:60-8; quiz 8-9.

15. Mystakidou K, Tsilika E, Parpa E, Galanos A, Vlahos L. Psychometric properties of the Impact of Event Scale in Greek cancer patients. J Pain Symptom Manage. 2007;33:454-61.

16. Maslach C, Jackson S, Leiter M. The Maslach Burnout Inventory Manual. 1997. p. 191-218.

17. Anagnostopoulos F, Papadatou D. Factor analysis and internal consistency of the Maslach Burnout Inventory in a sample of nursing staff members. Psychologika Themata (Greece). 1992;5:183-202.

18. Preti E, Di Mattei V, Perego G, et al. The Psychological Impact of Epidemic and Pandemic Outbreaks on Healthcare Workers:
The Journal of Critical Care Medicine 2021;7(1) • 19

Rapid Review of the Evidence. Curr Psychiatry Rep. 2020;22:43.

19. Karanikola M, Giannakopoulou M, Mpouzika M, Kaite CP, Tsiaousis GZ, Papathanassoglou ED. Dysfunctional psychological responses among Intensive Care Unit nurses: a systematic review of the literature. Rev Esc Enferm USP. 2015;49:847-57.

20. Costa DK, Moss M. The Cost of Caring: Emotion, Burnout, and Psychological Distress in Critical Care Clinicians. Ann Am Thorac Soc. 2018;15:787-90.

21. Rachiotis G, Kourousis C, Kamilaraki M, Symvoulakis EK, Dounias G, Hadjichristodoulou C. Medical supplies shortages and burnout among greek health care workers during the economic crisis: a pilot study. Int J Med Sci. 2014;11:442-7.

22. Zimeras S, Chardalias K, Diomidous M. Epidemiological Analysis of the Covid-19 Epidemic in Greece. Stud Health Technol Inform. 2020;272:21-3.

23. Tortella-Feliu M, Fullana MA, Pérez-Vigil A, et al. Risk factors for post-traumatic stress disorder: An umbrella review of systematic reviews and meta-analyses. Neurosci Biobehav Rev. 2019;107:154-65.

24. Ozer EJ, Best SR, Lipsey TL, Weiss DS. Predictors of posttraumatic stress disorder and symptoms in adults: a metaanalysis. Psychol Bull. 2003;129:52-73.

25. Carvalho PMM, Moreira MM, de Oliveira MNA, Landim JMM, Neto MLR. The psychiatric impact of the novel coronavirus outbreak. Psychiatry Res. 2020;286:112902.

26. Lima CKT, Carvalho PMM, Lima I, et al. The emotional impact of Coronavirus 2019-nCoV (new Coronavirus disease). Psychiatry Res. 2020;287:112915.

27. Barello S, Palamenghi L, Graffigna G. Burnout and somatic symptoms among frontline healthcare professionals at the peak of the Italian COVID-19 pandemic. Psychiatry Res. 2020;290:113129.

28. Vahedian-Azimi A, Hajiesmaeili M, Kangasniemi M, et al. Effects of Stress on Critical Care Nurses: A National Cross-Sectional Study. J Intensive Care Med. 2019;34:311-22.

29. Wakhid A, Hamid AYS. Family resilience minimizes posttraumatic stress disorder: A systematic review. Enferm Clin. 2020;30 Suppl 3:1-5.

30. Ntantana A, Matamis D, Savvidou S, et al. Burnout and job satisfaction of intensive care personnel and the relationship with personality and religious traits: An observational, multicenter, cross-sectional study. Intensive Crit Care Nurs. 2017;41:11-7.

31. Kousloglou S, Mouzas O, Nnotis K, Roupa Z, Vasilopoulos A, Angelopoulos N. Insomnia and burnout in Greek Nurses. Hippokratia. 2014;18:150-5.

32. Salpigktidis, II, Paliouras D, Gogakos AS, et al. Burnout syndrome and job satisfaction in Greek residents: exploring differences between trainees inside and outside the country. Ann Transl Med. 2016;4:444. 
[1] Gender:

$\square$ Male

$\square$ Female

[2] Age:

[3] Family status:

$\square$ Single

Married

Widow/er

Separated

$\square$ Divorced

[4] Do you have children?

$\square$ Yes

$\square$ No. If Yes, how many?

[5] Do you live:

$\square$ Alone

$\square$ With others

[6] Highest attained education level:

$\square$ High school
$\square$ Technological educational institute
$\square$ University
$\square$ Master's
$\square$ PhD

[7] When did you get your degree? Year

[8] What is your specialty?

$\square$ Physician

$\square$ Nurse

$\square$ Other - Which?

[9] Which department do you work in:

How long have you been working in total (in months)?

[10] In this profession:

[11] In the same hospital:

[12] In the same department:

[13] What led you to choose your profession? (choose one):

$\square$ My desire to help people

$\square$ The increased likelihood of finding a job later

$\square$ The influences I received from the environment

$\square$ I happened to be in this profession by chance 